\title{
Phase I studies: the role of publicly funded academic-healthcare partnerships
}

\author{
Nathan J Brendish clinical research fellow, Diane F Gbesemete clinical research fellow, Hans de \\ Graaf clinical research fellow, Christopher J Edwards director, Saul N Faust director
}

Southampton National Institute for Health Research Wellcome Trust Clinical Research Facility, University Hospital Southampton NHS Foundation Trust, Southampton General Hospital, Southampton SO16 6YD, UK

Emanuel and colleagues' meta-analysis found that phase I studies are very safe. ${ }^{1}$ However, the accompanying editorial mentioned that phase I studies are a "secret realm" and that "most studies are conducted outside academic medical centres at private facilities run by pharmaceutical companies or contract research organisations."

After a major safety problem at a commercial phase I facility in $2006,{ }^{3}$ the UK Medicines and Healthcare products Regulatory Agency (MHRA) introduced an accreditation scheme to enhance phase I safety standards in the UK. There are now four publicly funded phase I accredited clinical research facilities in the UK that have been open to non-commercial facilities since 2013 . These include one university-hospital partnership (the Southampton National Institute for Health Research (NIHR) Wellcome Trust Clinical Research Facility) funded by the National Institute for Health Research. This provides an environment for early phase studies within the heart of an acute NHS hospital along with academic input from local university investigators. Phase I studies in patient populations and healthy volunteers are a crucial part of drug development. The UK Department of Health NIHR experimental medicine infrastructure has created a setting that delivers industry and publicly funded phase I trials while allowing recruitment from the entire UK population.

Competing interests: NJB, HdG, and DFG are clinical research fellows and CJE and SNF the directors of the Southampton NIHR Wellcome Trust Clinical Research Facility.

1 Emanuel EJ, Bedarida G, Macci K, et al. Quantifying the risks of non-oncology phase I research in healthy volunteers: meta-analysis of phase I studies. BMJ 2015;350:h3271. (26 June.)

2 Kimmelman J. The secret realm of phase I trials in healthy volunteers. BMJ 2015;350:h3444. (26 June.)

3 Suntharalingam G, Perry MR, Ward S, et al. Cytokine storm in a phase 1 trial of the anti-CD28 monoclonal antibody TGN1412. N Engl J Med 2006;355:1018-28.

Cite this as: BMJ 2015;351:h3889

๑) BMJ Publishing Group Ltd 2015 